\title{
A 10-Day Binary System Orbiting the Be Star 66 Oph
}

\author{
S. Štefl, P. Hadrava \\ Astronomical Institute, Academy of Sciences, CZ-251 65 Ondřejov, \\ Czech Republic \\ D. Baade
}

European Southern Observatory, Karl-Schwarzschild-Str. 2, D-85748 Garching bei München, Germany

Th. Rivinius, M. Maintz, and O. Stahl

Landessternwarte Königstuhl, D-69117, Heidelberg, Germany

\begin{abstract}
Oph}$ is a multiple system with a close binary orbiting the B2Ve primary Be star. The binary parameters were derived by two independent methods and spectra of components BA and BB were separated by Fourier disentangling.
\end{abstract}

66 Oph (HR 6712, HD 164284, B2 Ve) belongs to the few bright Be stars observable from both the northern and the southern hemisphere. Its detailed history of emission activity was summarized by Floquet et al. (2002). They also derived fundamental parameters of the Be star and periods and modes of its non-radial pulsations. Up to now it was believed that $66 \mathrm{Oph}$ is a single $\mathrm{Be}$ star.

Our first observations disclosed variable and relatively narrow $\mathrm{Mg}$ II 4481 line components superimposed on the broad Be star profile. Similar features observed in another Be star, $o$ And, were interpreted as evidence of a multiple stellar system. In order to verify this suspicion, we monitored the star with the Heros echelle spectrograph $(R=20000,3450-8620 \AA)$ attached to the Ondřejov $2 \mathrm{~m}$ telescope between August 2000 and September 2002. 71 Ondřejov spectra were analyzed together with three more Heros spectra obtained at the Calar Alto 1.23m telescope in Aug-Sep, 1998.

Radial velocities for the two components $\mathrm{BA}$ and $\mathrm{BB}$ were determined by fitting Gaussian profiles interactively to the narrow components in the region of the Mg II 4481 and Mg I 5183 lines. Mean weighted values were calculated. A time series analysis using the MIDAS TSA package yielded the period $\mathrm{P}=(10.780$ $\pm 0.024) \mathrm{d}$.

We used two independent methods to derive the orbital parameters of the BA+BB binary within 66 Oph:

i) Solution of the RV curve with the help of the Fotel code (Hadrava, 2002).

ii) Fourier disentangling of line profiles using the KoREL code (Hadrava, 1997). Narrow regions around $\lambda \lambda$ 4481, 5167 and $5183 \AA$ sampled with steps below $1 \mathrm{~km} / \mathrm{s}$ per bin were disentangled simultaneously assuming a two-component 
system. A possible contribution of the broad Be component was treated as a continuum shift only.

Because the eccentricity converged to zero below the 0.001 limit for both methods, a circular orbit was adopted and the periastron longitude was fixed at 90 degrees. The resulting other orbital parameters are listed in Table 1.

Table 1. Parameters of the 66 Oph BA+BB binary derived by solving of the RV curve and by Fourier disentangling. No errors are provided by the KOREL code.

\begin{tabular}{lll}
\hline Parameter & RV curve solution & Fourier disent \\
\hline Period [day] & $10.779 \pm 0.012$ & 10.780 \\
periastron epoch -2400000 & $52010.19 \pm 0.04$ & 52010.19 \\
primary RV semiamp. $[\mathrm{km} / \mathrm{s}]$ & $46.3 \pm 1.7$ & 48.4 \\
secondary RV semiamp. $[\mathrm{km} / \mathrm{s}]$ & $75.1 \pm 6.4$ & 76.6 \\
mass ratio & $0.62 \pm 0.03$ & 0.63 \\
\hline
\end{tabular}

The BA and BB spectra were reconstructed in the spectral intervals 4468 4486 and $4545-4610 \AA$ and compared with a synthetic spectrum $\left(T_{\text {eff }}=10000\right.$ $\mathrm{K}, \log g=4)$ and observed template spectra. The spectra of both 66 Oph BA and $\mathrm{BB}$ are dominated by singly ionized metals, mainly Fe II, Ti II and $\mathrm{Cr}$ II. A very preliminary guess, using also the mass ratio and the $\mathrm{Mg}$ II / $\mathrm{Mg}$ II line ratio, indicates that the BA component is of late-B to early-A spectral subtype and the $\mathrm{BB}$ component is later by about one spectral class.

The FWHM was determined separately for the spectra of the A (Be), BA and $\mathrm{BB}$ components. $v \sin i=244 \mathrm{~km} / \mathrm{s}$ was derived for the Be component from the He I 4471 line. Our estimates for the BA and BB components are of the same order as the spectral resolution of the HERos spectra, it is $15-17 \mathrm{~km} / \mathrm{s}$. Data with higher resolution are needed to get more significant values from the minima of the Fourier transform and to test if the BA+BB binary affects the Be star solution.

The absence of detectable RV variations of the primary Be star indicates that the period of the $\mathrm{A}+(\mathrm{BA}+\mathrm{BB})$ system is at least of the order of tens of years and the close binary system does not affect the pulsation properties of the Be star. However, the $\mathrm{BA}$ and $\mathrm{BB}$ components make a non-negligible contribution to the Balmer discontinuity of the whole system and may cause a systematic error in the determination of the physical parameters of the Be star by Floquet et al. (2002). Moreover, the variable metallic lines in the wings of the photospheric lines of the Be star need to be masked off in analyses of pulsation properties of the Be star.

\section{References}

Floquet, M., Neiner, C., Janot-Pacheco, E., Hubert, A.-M., Jankov, S., Zorec, J., Briot, D., Chauville, J., Leister, N.V., Percy, J.R., Ballereau, D., Bakos, A.G. 2002, A\&A 394, 137

Hadrava, P. 1997, A\&AS 122, 581

Hadrava, P. 2002, http://www.asu.cas.cz/ had/fotel.html 